Revista Eletrônica de Ciência Administrativa (RECADM) - ISSN 1677-7387

Faculdade Cenecista de Campo Largo - Coordenação do Curso de Administração

v. 3, n. 2, nov./2004 - http://revistas.facecla.com.br/index.php/recadm/

\title{
LA CALIDAD DE SERVICIO: UNIDIMENSIONALIDAD VS. MULTIDIMENSIONALIDAD (UNA ESCALA ESPECIFICA PARA EL SECTOR DE BANCA COMERCIAL)
}

Santiago Merino, José.

Dr. En CC. Económicas y Empresariales

Catedrático E.U. Comercialización e Investigación de Mercados

Universidad Complutense de Madrid

\section{Resumen:}

El presente artículo pretende arrojar luz sobre la controversia suscitada entre los dos principales enfoques o paradigmas de medición de la Calidad de Servicio (Parasuraman, Zeithaml y Berry Cronin y Taylor), proponiendo una diferente escala de medida del constructo para el sector de banca comercial, fruto de la replicación "ex novo" de la investigación de PZB, con un enfoque de convergencia o triangulación metodológica y de técnicas de análisis.

Se enfrentan los dos modelos dominantes en un intento de confirmación/refutación de la forma de operacionalizar el constructo "calidad de servicio" en cada uno de ellas, así como de la unidimensionalidad del mismo frente a la multidimensionalidad.

Supone, asimismo, el desarrollo de las sugerencias de los citados investigadores en el sentido de ampliar el domino de los contenidos de la calidad de servicio a los productos/servicios, precio e imagen de las entidades, y de analizar con mayor profundidad las relaciones de la calidad con la satisfacción y con la lealtad, buscando mejores predictores de la última con la integración de aspectos actitudinales y comportamentales en su operativización.

Entre los principales resultados destaca la falta de adecuación del modelo explicativo de la calidad de servicios basado en las divergencias, siendo la forma de opracionalización del constructo que mejor se ajusta a los datos aquella que se basa sólo en la percepción del desempeño, cuyos resultados obtienen mejor ajuste, fiabilidad y validez tanto convergente como predictiva. Respecto a la estructura dimensional se confirma la multidimensionalidad del constructo, abogando por una estructura compleja superior a los 25 factores, que en la práctica implica su reducción a una sola (magnitud escalar) que puede ser expresada en un índice de calidad, lo que facilita su utilización en el proceso de toma de decisiones.

Palabras clave: Calidad de Servicio, Escalas de Medida, Banca

Esta pesquisa pretende iluminar a controvérsia suscitada entre os dois principais enfoque ou paradigmas da mediçao da Qualidade do Serviço (Parasuraman, Zeithml y Berry -Cronin y Taylor), propondo uma escala diferente de mediçao do construto para o setor da banca comercial, conseqüência da replicaçao "ex novo" da investigaçao do PZB, com um enfoque de convergencia ou triangulaçao metodológica e de técnicas de análise.

Confrontam-se os dois modelos dominantes numa tentativa de confirmaçao/refutaçao do jeito de operacionar o construto "qualidade do serviço" em cada um deles, assim como da unidimensionalidade do mesmo perante a multidimensionalidade.

Do mesmo modo supoe o desenvolvimento das indicaçoes dos referidos pesquisadores para incrementar o dominio dos conteudos da qualidade do serviço aos produtos/serviços, preços e imagem das entidades, e analisar com maior profundidade as relaçoes da qualidade com a satisfaçao e com a lealdade, procurando melhores preditores da ultima com a integraçao de aspectos atitudinais e comportamentais na sua operatividade.

Keys words:Qualidade do Serviço, Escalas de mediçao, Banca. 
Revista Eletrônica de Ciência Administrativa (RECADM) - ISSN 1677-7387

Faculdade Cenecista de Campo Largo - Coordenação do Curso de Administração v. 3, n. 2, nov./2004 - http://revistas.facecla.com.br/index.php/recadm/

\section{INTRODUCCIÓN}

la Calidad de Servicio ha sido utilizada en el sector bancario como una de las estrategias de diferenciación con mayor potencial de éxito. La aparente relación de la misma con los costes, la satisfacción de los clientes y la fidelidad ha hecho que tanto la una como las otras se hayan convertido no sólo en un arma estratégica para la empresa, sino también como un interesante tópico a investigar.

En este marco general dos tipos de consideraciones nos llevan a abordar dicho contenido en el presente trabajo: una de orden académico y otra en el ámbito del management empresarial.

Respecto de la primera, son innumerables los estudios académicos a que ha dado lugar, habiendo surgido diversos modelos, orientaciones o escuelas que están en permanente controversia. Entre ellos, el más ampliamente difundido y soportado es el propuesto por Parasuraman, Zeithaml y Berry (1988), en adelante PZB. Sin embargo, dicho modelo ha sido y es ampliamente cuestionado por diversos autores (Carman, 1990; Teas, 1993; Cronin y Taylor, 199294) ya por la utilización del paradigma de las divergencias en el campo de la calidad de servicio, ya por la utilización de las expectativas y su interpretación, ya por la multidimensionalidad y su generalización a los diferentes sectores de servicios.

Revisada la bibliografía, constatada la ardua controversia existente entre los dos principales enfoques o paradigmas de medición de la calidad de servicio: SERVPERF (Cronin y Taylor) y SERVQUAL (Parasuraman, Zetihaml y Berry) y analizados sus argumentos, se concluye la necesidad de investigar modelos de calidad que integren calidad, satisfacción del cliente, valor del servicio, así como su relación no sólo con las intenciones de compra sino también con la fidelidad concretada en comportamientos y de examinarlos a niveles particulares y globales de análisis.

Es el anterior, pues, el marco de partida de nuestra investigación, cuya pretensión es arrojar luz a la controversia suscitada, en especial en lo concerniente al incremento de la validez predictiva de los modelos de toma de decisiones del consumidor en el ámbito particular y específico de los servicios bancarios.

En el ámbito no académico, en particular en el de management de servicios, los estudios realizados y aplicaciones analizadas también han sido numerosos, por lo general trasladando el modelo más ampliamente difundido (SERVQUAL), con ligeros retoques y modificaciones, a la captación de la información, el análisis de la misma y como soporte coherente en el diseño de estrategias, establecimiento de planes de mejora y control de los resultados obtenidos.

Sin embargo, en dichos ámbitos empresariales y en especial en las empresas proveedoras de la información de la calidad percibida por el cliente (institutos de investigación, consultoras, etc...) se enfrentan permanentemente a un dilema respecto a la operatividad del modelo empleado: o un proceso de recogida y análisis de la información costoso derivado del número de atributos que contienen las escalas y las diversas operaciones que recaen sobre la misma (puntuaciones de calidad percibida, puntuaciones de calidad esperada, puntuaciones de importancia, determinación de niveles dentro de los atributos, establecimiento de la importancia discriminante...) y que obliga a sacrificar el $n^{\circ}$ de elementos (o a la utilización de una escala relativamente corta de 22 items, como la SERVQUAL) en beneficio de las operaciones a realizar sobre las mismas o, por el contrario, a la utilización de escalas más amplias (con mayor $n^{\circ}$ de atributos), más concretas y adaptadas al sector o servicio de que se trate y que permitan establecer con mayor concreción y especificidad los objetivos de mejora y, paralelamente, poder reducir fácilmente la información de calidad de servicio a pocos o un solo indicador, con sacrificio de las operaciones a realizar sobre los atributos. 
Revista Eletrônica de Ciência Administrativa (RECADM) - ISSN 1677-7387

Faculdade Cenecista de Campo Largo - Coordenação do Curso de Administração

v. 3, n. 2, nov./2004 - http://revistas.facecla.com.br/index.php/recadm/

\section{OBJETIVOS}

Dos grandes líneas nos han movido a la realización de este trabajo: por una parte, la evidente necesidad en el sector bancario de disponer de una herramienta específica, fiable, operativa, dinámica y flexible para medir la calidad de servicio en las entidades financieras; por otra, analizar en profundidad los aspectos que componen la calidad de servicio, evaluar los diferentes instrumentos de medida (SERVQUAL, SERVPERF) y proponer, en su caso, una escala de medición específica para las entidades financieras

\section{HIPÓTESIS}

Del anterior planteamientos, una vez realizado el Estudio Exploratorio Previo y analizado el estado de la cuestión en el ámbito académico, deducimos las siguientes hipótesis que serán objeto de contraste a lo largo del desarrollo de la investigación:

\section{a) Respecto a la Validez de los diferentes constructos de Calidad de Servicio :}

H1: La Calidad de Servicio Bancario es un constructo multidimensional, cuyo número de dimensiones sobrepasa las actualmente contempladas en el estudio de la calidad en el sector servicios (5), y permite una aplicación dual: por una parte su reducción forzada a un solo indicador de percepción de la Calidad de Servicio Global, equivalente al constructo unidimensional; por otra, la aplicación extensa (con la relación de items definitorios del servicio que se estime conveniente por cada entidad y en cada momento concreto) que permita la captación de información concreta y precisa que fundamente el establecimiento de objetivos de mejora, los objetivos de formación y el control de ambos.

b) Respecto a la forma de medir la Calidad de Servicio Bancario:

H2: La medición basada exclusivamente en el rendimiento (Calidad de Servicio Percibida) es más adecuada que la basada en el rendimiento menos las expectativas (Calidad de Servicio Percibida-Expectativas) .

H3: La Calidad de Servicio Percibida es un constructo multidimensional, de un gran número de factores, por lo que su utilización resulta harto dificultosa y poco operativa, aconsejando la búsqueda de formulas más sencillas y parsimoniosas de medir el constructo.

H4: La Calidad de Servicio Percibida, evaluada globalmente, es mejor indicador de la calidad percibida que cualesquiera otros basados en la dimensionalidad del constructo.

\section{METODOLOGÍA}

Se aborda el objeto de estudio con un enfoque multimétodo de fases, en cuya primera fase cualitativa se explora, descubre y contextualiza el problema de la calidad de servicio bancario y sirve, además, para plantear hipótesis, basar la confección del instrumento de medida y, posteriormente, en una segunda fase, cuantitativa, comparar y generalizar los resultados de la primera (integración combinatoria), intentando analizar y valorar la confluencia/divergencia de resultados de ambos enfoques (integración convergente), sin partir de supuestos teóricos apriorísticos dominantes en el ámbito de la calidad de servicio, sino aceptando lo que confluye con la teoría dominante y lo que discrepa de la misma, facilitando, con ello, el planteamiento de hipótesis alternativas que, en la fase posterior cuantitativa, serán contrastadas con resultados de aval o refutación.

\section{Fase 1: Estudio Previo Cualitativo}


Revista Eletrônica de Ciência Administrativa (RECADM) - ISSN 1677-7387

Faculdade Cenecista de Campo Largo - Coordenação do Curso de Administração v. 3, n. 2, nov./2004 - http://revistas.facecla.com.br/index.php/recadm/

Esta fase se corresponde con una investigación exploratoria, de tipo cualitativo, consistente en la realización de 16 reuniones de grupo no dirigidas, con clientes de entidades bancarias de la Comunidad de Madrid que cubrían un amplio espectro no sólo en cuanto a nivel cultural, económico, clientes de diferentes entidades y diferente nivel de bancarización

De las transcripciones de tales reuniones se realizó un análisis de contenido temático de las verbalizaciones obtenidas y registradas, de donde resultan los aspectos más relevantes, los aspectos concretos, que dan lugar a una primera categorización o agrupación en factores. A partir de aquí, se agruparon en categorías más generales (categorías operativas) y posteriormente en dimensiones analíticas (Ver Figura $n^{0} 1$, del Anexo). En cada uno de los factores se recogen "verbatin" significativos que ilustran aquellos indicadores de expectativas y percepciones consideradas en cada una de las categorías y dimensiones.

\section{Fase 2: Construcción de la Escala de Calidad de Servicio Bancario}

Esquemáticamente, para la construcción de la Escala de Calidad de Servicio Bancario, se ha seguido el siguiente procedimiento:

1) Creación de una Escala Preliminar según las especificaciones

2) Aplicación de la Escala Preliminar a una muestra apropiada.

3) Análisis de la forma preliminar de la escala.

4) Revisión subsiguiente y elaboración de la escala final-definitiva.

5) Aplicación de la Escala Definitiva a una muestra de la misma población.

6) Validación de la Escala Definitiva.

Los pasos 1 a 6 anteriores se omiten, por razones de espacio en este artículo.

\section{Fase 3: Estudio Empírico}

Se seleccionó una muestra de 1198 individuos (mayores de 18 años y usuarios bancarios de la C.A. Madrid), estratificada por tipo de hábitat, sexo y edad, y por sistema polietápico de rutas aleatorias. Para un nivel de confianza de 2 sigmas, el error muestral máximo resultante, para los datos globales, sería de +/- 2,8 \% y para el supuesto más desfavorable de $p=q=50 \%$. Aplicando la corrección por utilizar el sistema polietápico y el correspondiente a la utilización de variables multicotómicas, el error máximo corregido es de +/-4,30.

El cuestionario recababa información sobre comportamientos: (frecuencia de visita a la oficina bancaria, entidades con las cuales se trabaja, antigüedad con las mismas, entidad principal, productos/servicios utilizados, ruptura total y parcial con entidades, motivos de ruptura, acciones de retención percibidas, ...) y actitudes: ( grado de satisfacción con entidades con las que se trabaja, intenciones de ruptura total y parcial y sus motivos, entidades recomendadas...así como escala de valor en la elección de entidad bancaria, y evaluación de calidad de servicio de la entidad principal).

\section{RESULTADOS}

\subsection{ESCALA DEFINITIVA}

La escala definitiva, resultante del proceso de depuración anteriormente explicitado, está integrada por un conjunto de 56 variables que representan atributos de la percepción de calidad del servicio bancario. Dicha escala posee, a nuestro juicio, las siguientes características metodológicas: es específica, esto es, diseñada a medida del sector bancario; todas las variables se enuncian en sentido positivo; cada variable recoge la información en una escala de intensidad 
Revista Eletrônica de Ciência Administrativa (RECADM) - ISSN 1677-7387

Faculdade Cenecista de Campo Largo - Coordenação do Curso de Administração

v. 3, n. 2, nov./2004 - http://revistas.facecla.com.br/index.php/recadm/

tipo Likert, sencilla, de 5 puntos que se adapta mejor a la estructura mental de los entrevistados que la de 7 puntos, discrimina suficientemente entre las diversas posiciones y es suficientemente sensible.

\subsubsection{Fiabilidad de la Escala Definitiva}

Para proceder al cálculo de la fiabilidad de la Escala Definitiva de C.S.B. se han empleado varios criterios: el del coeficiente alpha de Cronbach (1951) y el de las dos mitades.

En la tabla $\mathrm{n}^{0} 1$ observamos los datos correspondientes a la fiabilidad de varios formatos de escala, según los criterios que acabamos de describir. La escala denominada "Total" o Global es aquella que abarca todos los items empleados para medir la C.S.B. También consideramos otras que incluyen por separado los contenidos de las dimensiones establecidas en la construcción preliminar de la escala. El coeficiente alpha muestra las correlaciones de cada elemento con la suma total de la escala y con la media total de la escala.

Tabla n¹: Fiabilidad de las diversas alternativas de medida de la C.S.B.

\begin{tabular}{|c|c|c|c|}
\hline & \multicolumn{2}{|c|}{$\begin{array}{c}\text { Coeficiente } \\
\text { FORMA DE MEDIR EL }\end{array}$} & \multicolumn{2}{|c|}{$\begin{array}{c}\text { Coeficientes Dos } \\
\text { mitades }\end{array}$} \\
\cline { 3 - 4 } CONSTRUCTO C.S.B. & Alfa de Cronbach & $1^{\text {a } \text { mitad }}$ & $\begin{array}{l}2^{0} \\
\text { mitad }\end{array}$ \\
\hline $\begin{array}{c}\text { C.S. PERCIBIDO (RESULTADO) } \\
\text { C.S. (E- P) (EXPECTATIVAS } \\
\text { MENOS PERCEPCION DE } \\
\text { RESULTADO) }\end{array}$ & 0,96 & 0,93 & 0,92 \\
\hline $\begin{array}{c}\text { C.S. (E - P) X I (EXPECTATIVAS } \\
\text { MENOS RESULTADO x } \\
\text { IMPORTANCIA) }\end{array}$ & 0,94 & 0,91 & 0,88 \\
\hline
\end{tabular}

Las tres formas de operacionalizar el constructo C.S.B. y sus tres escalas de medida resultan ser, pues, fiables, como demuestran los correspondientes valores alpha. Sin embargo, puesto que la forma de hacer operativo el constructo basado solo en la percepción de resultado obtiene mejores índices de homogeneidad (además de validez, según luego veremos) determinados análisis estadísticos están referidos sólo a esta última.

Para asegurarnos más de la homogeneidad de la escala elegida 'PERCEPCION DE RESULTADO' ante la posible objeción de que su alta fiabilidad puede venir en gran medida determinada por el amplio número de elementos $(n=56)$, más que por una verdadera fiabilidad, se han calculado índices de fiabilidad parciales para cada dimensión resultante del análisis factorial exploratorio, siendo sus resultados igualmente positivos, a excepción de las dos últimas dimensiones que sólo tienen dos elementos, como observamos en la tabla $n^{\circ} \quad 2$, siguiente:

Tabla n² Indices de Fiabilidad / Homogeneidad de la Escala Definitiva

Alpha 
Revista Eletrônica de Ciência Administrativa (RECADM) - ISSN 1677-7387

Faculdade Cenecista de Campo Largo - Coordenação do Curso de Administração v. 3, n. 2, nov./2004 - http://revistas.facecla.com.br/index.php/recadm/

\begin{tabular}{|l|c|}
\hline \multicolumn{1}{|c|}{ Total Escala } & \\
\hline \multicolumn{1}{|c|}{ Dimensiones } & 0.96 \\
\hline F1: Profesionalidad / Trato / Amabilidad & 0.94 \\
\hline F2: Oficinas / Instalaciones & 0.85 \\
\hline F3: Imagen / Productos y Servicios) & 0.83 \\
\hline F4: Rapidez & 0.82 \\
\hline F5: Información / Transparencia & 0.78 \\
\hline F6: Eficacia / Fiabilidad & 0.74 \\
\hline F7: Funcionamiento Cajeros y Equipos & 0.54 \\
\hline F8: Flexibilidad & 0.40 \\
\hline
\end{tabular}

En resumen, basándonos en los resultados derivados del empleo de criterios estadísticos de evaluación de la fiabilidad, podemos afirmar que la escala construida para medir la C.S.B. es altamente fiable, tiene consistencia interna, pues los valores obtenidos siempre exceden del nivel 0.7 (a excepción de las dimensiones 7 y 8 que difícilmente pueden conseguir el mínimo al estar reducido su contenido a sólo dos items).

\subsubsection{Validez de contenido}

La escala de Calidad de Servicio Bancario que presentamos consigue una alta validez de contenido, en nuestra opinión, por las siguientes razones:

1) Se intenta una definición conceptual de la calidad de servicio bancario recurriendo a la realización de un estudio exploratorio entre usuarios, lo que nos ha permitido generar una amplia relación de ítems que representan todas las dimensiones relevantes del fenómeno.

2) Se ha revisado y analizado la literatura y los planteamientos teóricos, las experiencias llevadas a cabo, las críticas y sugerencias a que han dado lugar, para intentar contemplar todos los atributos a medir y la mejor forma de hacerlo en el sector específicamente bancario. En este sentido se amplia el dominio a los productos y servicios bancarios (sugerencia de PZB, 1994), como se constata en nuestro estudio exploratorio previo.

3) Se han analizado los items mediante técnicas estadísticas (Véanse los resultados que se refieren a "Validez de constructo"

4) Se ha aplicado la escala a diferentes muestras de usuarios bancarios típicos. Una para la construcción de la escala preliminar y otra diferente para validar la escala definitiva.

\subsubsection{Validez de concepto (constructo)}

Del examen de la literatura sobre la calidad de servicio y, en concreto sobre la calidad de servicio bancario, se deducen una serie de supuestos sobre las variables y sus interrelaciones, que nos llevan a formular hipótesis apriorísticas y a contrastarlas empíricamente. En nuestro caso existen varias hipótesis previas sobre el $n^{\circ}$ de factores o dimensiones que integran el constructo "Calidad de Servicio Bancario":

- La "calidad de servicio bancario" es un constructo multidimensional de 5 dimensiones, basado en el modelo de discrepancias de PZB(88). Dicha hipótesis plantea que los métodos alternativos de medición empleados representan dimensiones distintas que no convergen para medir una única variable subyacente.

- La "calidad de servicio bancario" es un constructo unidimensional, conforme a los hallazgos de Cronin y Taylor $(92,94)$ y, por tanto, sus múltiples procedimientos de medición convergen para representar una construcción 
Revista Eletrônica de Ciência Administrativa (RECADM) - ISSN 1677-7387

Faculdade Cenecista de Campo Largo - Coordenação do Curso de Administração

v. 3, n. 2, nov./2004 - http://revistas.facecla.com.br/index.php/recadm/

única. En tal caso, sus diversos indicadores son piezas de información redundante o paralelas.

- La "calidad de servicio bancario" es un constructo multidimensional, de más de 5 dimensiones, como sugiere el análisis exploratorio de nuestros datos o bien un constructo unidimensional.

La validez de constructo se evalúa, pues, investigando qué cualidades mide la escala de C.S.B., viendo si confirman o niegan las hipótesis precedentes sobre calidad de servicio. Ahora bien, si las definiciones operativas previas coincidieran sería relativamente fácil establecer si se produce validez. Pero cuando, como en el constructo que nos ocupa, no coinciden e incluso hay discrepancia abierta entre los investigadores, la tarea de las nuevas aportaciones se hace especialmente delicada, pues si el investigador pretende conseguir la mayor validez de constructo ha de hacer un previo cálculo de los trabajos que avalan -o mejor, coinciden- las posturas discrepantes si quiere ver incrementadas la validez "convergente" de la escala que propone. dimensional.

Para evaluar dicha validez de constructo hemos procedido a realizar un análisis

Las 56 variables (items) de la Escala Definitiva, operacionalizada en sus diversas formas como "percepción de resultado" (C.S.Percibida), "percepción de resultado menos expectativas" (C.S.P-E) y ésta última ponderada por la importancia (P-E)xI, han sido analizadas factorialmente usando el método de Componentes Principales.

En los siguientes pasos sólo haremos referencias a los análisis estadísticos referidos a la forma de operacionalizar el constructo que mayor fiabilidad ha obtenido y cuya matriz de correlaciones es la más idónea. Se trata de la Percepción de Resultado (C.S. PERCIBIDO).

Resultan 9 factores o dimensiones que explican el 56,3 \% de la varianza. En esta solución, sin rotar, se observa que casi todas las variables cargan en gran medida en el Factor 1, con un recorrido de 0,75 a 0,36. Sólo los items CSR34 (ausencia de colas), y CSR34 (Esperas cortas...) cargan más en otras dimensiones que en la principal.

Del peso de cada variable en los factores y del conjunto de las mismas se deduce la conceptualización de cada dimensión, como se aprecia en la tabla siguiente:

Tabla nº 4: Explicación de los Factores

\begin{tabular}{|c|c|c|}
\hline Factor & Explicación & $\begin{array}{c}\text { Varianza } \\
\text { explicada (\%) }\end{array}$ \\
\hline FACTOR I: & Profesionalidad - Trato - Amabilidad & 20 \\
\hline FACTOR II & Oficinas - Instalaciones & 9,6 \\
\hline FACTOR III & Factores de Imagen y Oferta Comercial & 5,9 \\
\hline FACTOR IV & Rapidez de Servicio & 5,21 \\
\hline FAC TOR V & Información - Transparencia & 4,98 \\
\hline FACTOR VI & Eficacia - Fiabilidad & 4,82 \\
\hline FACTOR VII & Funcionamiento de Cajeros y Equipos & 3,12 \\
\hline FACTOR VIII & Flexibilidad & 1,85 \\
\hline \multicolumn{2}{|r|}{ Total... } & 54,48 \\
\hline
\end{tabular}

Se observa en la tabla $\mathrm{n}^{\circ} 5$ (Anexo) existe una cierta concurrencia de variables de contenidos dispares en un mismo factor, como el F-III: Imagen, Productos y Servicios, que aparecen separadas al incrementar el $n^{\circ}$ de factores. También se aprecia una separación de conceptos que intuitivamente deberían ir unidos, como las variables de Factor VI y las de Factor 
Revista Eletrônica de Ciência Administrativa (RECADM) - ISSN 1677-7387

Faculdade Cenecista de Campo Largo - Coordenação do Curso de Administração v. 3, n. 2, nov./2004 - http://revistas.facecla.com.br/index.php/recadm/

VII (Eficacia, Fiabilidad y Correcto funcionamiento de los equipos). Asimismo el Factor IX (Familiaridad) encaja perfectamente en el contenido del FI (Trato).

Sin ánimo de forzar con la lógica del "martillazo" el encuadre de los items en los factores, exploramos otras alternativas de factorización y rotación, basándonos, además, en la existencia de correlación entre los factores (ya señalada en los trabajos de Cronin y Taylor ( 92), PZB (92) y Bigné et alia (98), quienes sugieren la rotación OBLIMIN como alternativa a la VARIMAX. Sin embargo, este tipo de rotación, aunque metodológicamente más adecuada, produce una matriz de componentes de difícil interpretación. Es por ello que procedimos a un reajuste factorial en el sentido de reubicar las citadas variables en el factor que correspondería por lógica e intuición (ver solución de la tabla número 5)

De manera complementaria procedemos a la evaluación de la dimensionalidad a través del análisis factorial confirmatorio utilizando el procedimiento de máxima verosimilitud, ya utilizado en la construcción de la escala preliminar y cuyos resultados abogaban por una estructura compleja, superior a los 15 factores. En esta segunda fase de validación convergente ( y también de fiabilidad) obtenemos resultados similares: el $\mathrm{n}^{0}$ de dimensiones que requiere el modelo para que sea adecuado tiene una frontera en 25 factores (chi-cuadrado 484; g.l.=444; sig.. 0.165). Recordamos que la significación tiene que ser superior a 0.05 para poder rechazar la hipótesis nula de que el número de factores considerado es el adecuado a los datos. La hipótesis nula considera que el número de factores elegidos es correcto; por tanto, si la significación es menor que 0.05 deberemos considerar otro número de factores.

En este caso, como la significación comienza a ser mayor que 0.05 al solicitar 25 factores, no rechazamos la hipótesis nula y, por tanto, podemos considerar que el número supuesto de factores (25) es correcto.

Desde el punto de vista de la validez convergente, los resultados se aproximan más al modelo unidimensional de Cronin que considera el constructo "calidad de servicio" como una sola construcción mental subyacente, una actitud en la valoración del servicio, que al modelo multidimensional de PZB.

Comparando los resultados de la escala C.S.B. con los obtenidos por otros autores en este mismo sector, o en otro similar utilizando las escalas SERVQUAL o SERPERF, resulta que aquella obtiene incluso superiores niveles de fiabilidad $y$, por otra parte, explica similares niveles de la varianza del fenómeno "Calidad de Servicio", (ver Tabla nº 6 del Anexo).

El análisis confirmatorio que efectuamos revelará cuál de las cuatro estructuras propuestas es la mejor de todas las posibles, con lo cual quedaría demostrada la validez convergente de la escala C.S.B.

La validez convergente se determina por medio de un estadístico que refleja la bondad de ajuste de cada modelo con los datos, concretamente comparando la bondad de ajuste de los modelos representativos de 1, 5, 8 y 25 factores.

El primero de ellos supone que toda la varianza (excepto el error de medición) es explicada por una sola construcción subyacente, denominada "calidad de servicio bancario" (Cronin, 1992).

El modelo de 5 factores propone que la C.S.B. se representa a través de 5 dimensiones independientes, aunque relacionadas entre si.

También se analizan los modelos de 8 y 25 factores, alternativos a los anteriores.

Si el índice de bondad de ajuste del modelo multidimensional es superior al índice unidimensional, la validez convergente corresponderá a la escala propuesta conforme al criterio de 


\section{Revista Eletrônica de Ciência Administrativa (RECADM) - ISSN 1677-7387 \\ Faculdade Cenecista de Campo Largo - Coordenação do Curso de Administração v. 3, n. 2, nov./2004 - http://revistas.facecla.com.br/index.php/recadm/}

PZB; de lo contrario se confirmará la estructura de Cronin. Si ninguna de ellas obtiene un índice ajustado probaremos con las estructuras de 8 y 25 factores.

La información contenida en la Tabla $n^{\circ} 7$ (Anexo) revela que el modelo de 25 factores se ajusta mejor a los datos que los modelos de 8, 5 ó 1 factores.

Para la realización del análisis factorial confirmatorio mediante ecuaciones estructurales se empleó el paquete estadístico AMOS (Arbuckle, J.L., 1994).

Hemos ensayado los dos procedimientos básicos para la estimación de los parámetros con AMOS: el método de máxima verosimilitud (ML) y el método de los mínimos cuadrados no ponderados (ULS).

Con ambos se obtienen resultados similares: el modelo que obtiene el mejor ajuste es el de 25 factores, seguido del de 8 factores y del de 1 factor. El modelo de 5 factores, aun obteniendo un buen ajuste, es inferior al resto y, además, es el que más residuos deja sin explicar. Dichas medidas de ajuste se observan en la tabla nº 8 del Anexo.

En consecuencia, la jerarquía de los modelos que se ajustan a los datos es la siguiente:

$1^{\circ}$ Modelo de 25 factores.

$2^{\circ}$ Modelo de 8 factores

Prescindimos de los modelos de 5 factores y del unidimensional por MENOR ajuste, el primero de ellos por sus excesivos residuos y el segundo por moderado ajuste.

El mal ajuste que pudiera derivarse de la magnitud del determinante de la matriz de correlaciones (Chi cuadrado y su p-valor no significativo) y es común a los diversos modelos enfrentados, debido a la existencia de valores 'outliers' o a la desviación de la normalidad de alguna variable. No obstante, cuando utilizamos otros indicadores de ajuste que no sean tan sensibles a la normalidad multivariante, obtenemos un ajuste aceptable de los modelos, salvo el de 5 factores.

Otra alternativa para la comparación entre modelos de la Escala (modelo unidimensional, modelo de 5 factores de PZB y los multidimensionales de 9 y 25 factores de nuestras hipótesis) es la utilización de la técnica de "BOOTSTRAP" (repetición de muestras) (Linhart y Zucchini, 1986) ${ }^{1}$, indicada para la elección no de un modelo en términos absolutos, sino para la elección de un modelo entre varios enfrentados (Browne, 1982; Stine, 1989).

El conjunto de las discrepancias para cada modelo se reproduce en la tabla $\mathrm{n}^{\circ} 9$ del Anexo:, incluyendo los valores BCC, AIC y CAIC.

En nuestro caso, la menor discrepancia $(4512,2)$ se obtiene para el modelo de 25 Factores, confirmando la elección de este modelo al juzgar los criterios de BCC, AIC, y CAIC.

En suma, a diferencia de lo que ha venido sucediendo en la mayor parte de los trabajos realizados, en nuestra investigación se atisba empíricamente que la C.S.B. es una constructo multidimensional, con dudas bastante razonables para admitir que el número de dimensiones sea el especificado por el modelo mayoritariamente seguido en la literatura de calidad de servicios (las 5 de PZB) o para admitir el modelo alternativo unidimensional propuesto por Cronin. Los datos de nuestro estudio hacen que aboguemos por un modelo multidimensional de al menos 25 factores, lo que hace que, en la práctica de la gestión de management de servicios en el sector bancario,

\footnotetext{
${ }^{1}$ Linhart, H. Y Zucchini, W.(1986): Model selection. New York. Wiley.

${ }^{2}$ Browne, M.W. (1982): Covariances structures. En Hawking, D.M. (Ed.): ‘Topics in applied multivariate analysis. Cambridge Univ. Press, p. 72-141.

Stine, R. (1989): An introduction to bootstrap methods: Examples and ideas. Sociological Methods and Research, 18, 243-291.
} 
Revista Eletrônica de Ciência Administrativa (RECADM) - ISSN 1677-7387

Faculdade Cenecista de Campo Largo - Coordenação do Curso de Administração v. 3, n. 2, nov./2004 - http://revistas.facecla.com.br/index.php/recadm/

guiados por el principio de parsimonia, todo modelo superior a 10 factores desemboque en un modelo unidimensional, tal y como propone, por diferentes motivos, Cronin $(1992,1994)$.

\subsubsection{Validez Discriminante.}

Diferentes procedimientos se pueden emplear para determinarla. En ocasiones se lleva a cabo un análisis factorial exploratorio donde intervienen las escalas de medición de varios conceptos, en espera de que los items de escalas diferentes no pesen en el mismo factor. Otros autores se basan en el coeficiente phi de correlación entre los factores obtenidos a partir del análisis factorial confirmatorio (Batra y Ahtola, 1990). Este estadístico constituye un indicador del grado de separación entre dos factores o dimensiones. Un alejamiento de la unidad constituye un indicio de que los factores obtenidos son realmente distintas $y$, por tanto, existe validez discriminante. En nuestro caso, referido al modelo de 5 factores, las correlaciones entre los factores varían de un mínimo de 0,5 a un máximo de 0,88, que indican poca separación entre los factores o escasa validez discriminante. Estos coeficientes se reducen considerablemente a medida que se incrementa el número de factores, siendo preciso llegar al modelo de 25 factores para obtener coeficientes inferiores a 0,3 , que indicarían la existencia de validez discriminante 0 separación entre los factores.

\subsubsection{Validez de Criterio}

Los criterios se dividen generalmente en concurrentes y predictivos. Determinar qué es concurrente y qué es predictivo no siempre resulta fácil. Sin embargo, los criterios predictivos se reservan generalmente para pronósticos de largo alcance. De ello se deriva la distinción entre validez concurrente y validez predictiva. Cuando hay relación entre la variable que pronostica y la variable criterio medidas en el mismo momento, o bien cuando hay correlación entre dos medidas distintas del mismo fenómeno que se realizan simultáneamente, nos estamos refiriendo a la validez concurrente. Por otro lado, la validez predictiva mide la capacidad para anticipar características, fenómenos o comportamientos futuros. En este caso, si la escala de C.S.B. resultare con validez predictiva, podría utilizarse para predecir el comportamiento de lealtad de los usuarios bancarios respecto de las entidades con las que operan.

Sin embargo, debe quedar claro que una escala que tenga validez concurrente no necesariamente tendrá validez predictiva. Una escala que mida la C.S.B. puede correlacionarse en alto grado con las intenciones de mantenimiento de la relación con una determinada entidad bancaria, pero es algo más difícil que correlacione con la conducta real futura del mismo sujeto. Las actitudes cambian con el tiempo y, por tanto, la relación que mantienen con la conducta será inferior a 1.

\subsubsection{Validez concurrente}

Por lo general, el criterio utilizado en la investigación de la validez concurrente es una medida sustituta, que reemplaza a otra más importante y también más difícil o gravosa de estimar.

En nuestro estudio se han considerado variable criterio la evaluación de la calidad global del proveedor principal y variables independientes, por una lado, la puntuación promedio resultante de las diversas formas de operacionalizar la CSB y, por otro, las puntuaciones factoriales de los diversos formatos de escala de C.S.B.

La mejor validez convergente se produce con la utilización de la escala en la que sólo se utiliza puntuaciones de desempeño, seguida por la que utiliza desempeño ponderado por importancia (Cronin, 1994) . Las formas propuestas por PZB resultan inferiores, siendo la más aceptable de ellas la de percepciones menos expectativas (Ver Tabla $n^{\circ} 10$ del Anexo). 
Revista Eletrônica de Ciência Administrativa (RECADM) - ISSN 1677-7387

Faculdade Cenecista de Campo Largo - Coordenação do Curso de Administração v. 3, n. 2, nov./2004 - http://revistas.facecla.com.br/index.php/recadm/

Además, dada la alta correlación entre Calidad de Servicio (Percibido-Esperado) y Calidad de Servicio (Percibido-Esperado ponderado por Importancia), de 0,978, por el principio de parsimonia, bien pudieran eliminarse las puntuaciones de importancia. Esta misma afirmación también es aplicable a la ponderación de C.S. Percibida por Importancia: Puesto que la correlación de C.S. Percibida $(0,474)$ es superior a la de C.S. (Percibida por Importancia) $(0,429)$ y entre ambas existe una alta correlación, por parsimonia se puede eliminar el factor de ponderación por las puntuaciones de importancia.

La validez concurrente deducida de la anterior tabla de correlaciones se corrobora al comparar los coeficientes beta de la regresión múltiple. En el modelo resultante sólo las formas de medición que no contemplan las expectativas, es decir C.S. Percibido y C.S. (Percibido por Importancia), resultan significativas, siendo la forma C.S. Percibida la que aporta una mayor contribución relativa (beta estandarizada) al modelo, y, por tanto, también obtiene el mayor coeficiente beta $(0,770)$ frente al mismo formato ponderado por importancia $(0,063)$. (Ver Tabla $\mathrm{n}^{\circ}$ 11, del Anexo)

\subsubsection{Validez Predictiva}

Una fórmula híbrida entre la validez de criterio concurrente y predictiva, ya ensayada en numerosas ocasiones (entre otros por PZB, Cronin y Taylor, Vázquez Casielles) consiste en medir concurrentemente, en el momento de aplicación de la escala, no la conducta futura sino la previsión de conducta futura de los usuarios a través de las intenciones de lealtad respecto de la entidad o de la previsión de otras conductas relacionadas como, p.e., la recomendación de la entidad a terceros, e incluso el nivel de satisfacción reportado por la entidad principal.

Considerando, pues, como variable dependiente la Intención de Ruptura (Total o Parcial) con la entidad principal proveedora financiera, resulta que el mejor predictor de la misma lo constituye la Satisfacción que ésta reporta (correlación de $-0,32$; pseudo $\mathrm{r} 2=24,8$; coeficiente $b=$ -1,54), seguida de la evaluación global de Calidad de Servicio de la misma entidad $(r=-0,28$; pseudo $r 2=-18,8$ y coeficiente $b=0,84$ ). La calidad de servicio, evaluada con la escala de C.S.B. y operacionalizada según diferentes alternativas, ofrece peores resultados.

Por tanto, la evaluación de los datos anteriormente referidos nos reafirma en las consideraciones realizadas anteriormente respecto a la unidimensionalidad del constructo de Calidad de Servicio Bancario frente a la multidimensionalidad, y, dentro de ésta, a la mejor predictividad de la solución de 25 factores (resultados del factorial de máxima verosimilitud, de los factoriales confirmatorio por ecuaciones estructurales y de la elección de modelos por repetición de muestras) que la de otro número de factores considerados.

En la tesitura de la multidimensionalidad del constructo C.S. Ios factores que resultarían significativos, tanto en las solución de 25 como en la de ocho factores, son : F1: Trato, Amabilidad; F3: Imagen de la Entidad y Oferta Comercial; F6: Eficacia, Fiabilidad, F8: Flexibilidad.

\section{RESUMEN, CONCLUSIONES E IMPLICACIONES}

\section{a) RESPECTO AL MODELO EXPLICATIVO DE LA CALIDAD DE SERVICIO BASADO EN LAS DIVERGENCIAS, Y SUS DIFERENTES FORMAS DE OPERACIONALIZACION:}

-El modelo basado en las divergencias entre el desempeño y las expectativas, si bien resulta ilustrativo y coherente en la práctica, no aparece refrendado por nuestros datos, por cuanto al escaso poder discriminante de las puntuaciones de expectativas se añade su nulo impacto sobre la satisfacción o lealtad, sin aportar explicaciones complementarias al modelo del simple desempeño, más sencillo en su utilización y viable en la captura de la información. 
Revista Eletrônica de Ciência Administrativa (RECADM) - ISSN 1677-7387

Faculdade Cenecista de Campo Largo - Coordenação do Curso de Administração v. 3, n. 2, nov./2004 - http://revistas.facecla.com.br/index.php/recadm/

-Comparados los resultados de las diferentes formas de operacionalizar el constructo Calidad de Servicio Bancario no podemos descartar completamente las formas C.S. $=P-E$ ó C.S.= $(P-E) x$ I, sin embargo la forma más sencilla y parsimoniosa, basada sólo en la percepción del desempeño o resultado obtiene mejor ajuste, fiabilidad, validez convergente y predictiva, en la aplicación de diferentes y alternativas técnicas de análisis efectuadas, razón por la cual se hace recomendable.

\section{b) RESPECTO A LA DIMENSIONALIDAD DEL CONSTRUCTO C.S.B.}

-Se confirma la multidimensionalidad del constructo Calidad de Servicio Bancario, con dudas bastante razonables para admitir que el número de dimensiones sea el especificado por el modelo mayoritariamente seguido en la literatura de calidad de servicios (las 5 dimensiones de Parasuraman, Zeithaml y Berry) o para admitir el modelo alternativo unidimensional de Cronin y Taylor). Utilizando el análisis factorial confirmatorio por máxima verosimilitud y comparación de modelos con diferentes número de factores mediante AMOS (ecuaciones estructurales) y 'bootstrap' (repetición de muestras), los resultados abogan por una estructura compleja, superior a los 25 factores, lo que hace que, en la práctica de management de servicios en el sector bancario, guiados por el principio de parsimonia, todo modelo superior a diez factores desemboque en un modelo unidimensional, tal y como proponen, por diferentes motivos Cronin y Taylor.

Ello implica la reducción o transformación de la calidad de una magnitud multidimensional a una magnitud escalar, que puede ser expresada en un índice, facilitando con ello el procedo de decisión .

No obstante, caso de considerarse conveniente, en algún caso, la utilización de la operacionalización multidimensional, es más recomendable utilizar 8 o 9 factores que los 5 propuestos por PZB.

\section{c) RESPECTO A LA ESCALA C.S.B. (CALIDAD DE SERVICIO BANCARIO) PROPUESTA.}

-La escala de Calidad de Servicio Bancario que proponemos no sólo utiliza items que describen características de calidad de servicio, sino otros relativos al producto, al precio y a la imagen de la entidad bancaria, con la pretensión de que proporcione mayor poder explicativo sobre la realidad del servicio bancario y mayor poder predictivo sobre la fidelidad.

La escala propuesta no sólo pretende ser un instrumento válido para diagnosticar o certificar el nivel de calidad en el sector bancario sino también un instrumento de búsqueda de elementos concretos para basar los programas de mejora y, conjuntamente con la satisfacción y otras variables, hacer un pronóstico de fidelidad a las entidades.

Las características metodológicas de la Escala C.S.B. se resumen en:

a) Es específica para el sector bancario: está diseñada a la medida del sector 'banca comercial' en que se desarrolla la investigación.

b) No contiene items de terminologías mezcladas; todos los items se enuncian en términos positivos.

c) No se solicitan puntuaciones de expectativas ni de importancia (aunque en el estudio sí han sido solicitados, para comparar las diferentes formas de operacionalizar el constructo)

d) La escala es altamente fiable (alpha $=0,96$ )

e) Consigue una alta validez de contenido, y una aceptable validez de constructo (validez de supuesto, validez convergente, y validez discriminante sólo en el modelo de 25 factores)

f) Aceptable validez de criterio, tanto concurrente como predictiva 
Revista Eletrônica de Ciência Administrativa (RECADM) - ISSN 1677-7387

Faculdade Cenecista de Campo Largo - Coordenação do Curso de Administração v. 3, n. 2, nov./2004 - http://revistas.facecla.com.br/index.php/recadm/

\section{IMPLICACIONES}

Diversas son las implicaciones a las que los resultados de este estudio dan lugar, tanto en el ámbito de la investigación académica como en la práctica de las gestión de la calidad. Algunas de ellas se refieren a continuación.

\section{a) En el ámbito de la Investigación Académica:}

- Las escalas generales de Calidad de Servicio encuentran difícil acomodo en su aplicación a los diferentes sectores. Se hace necesario construir escalas específicas de medida de la calidad en cada sector e incluso en cada tipo de empresa. Construir modelos explicativos y sistemas de medida generalizables es pretensión científica justificada, pero a menudo resultan no operativas en la práctica.

- Si bien el modelo de las divergencias resulta ilustrativo y coherente conceptualmente, cuando se amplía el modelo relacionándolo con conductas de fidelidad, con fines predictivos, el indicador de calidad de servicio global y el de satisfacción son más consistentes. Conviene, pues profundizar en el modelo de calidad y su potencial predictivo en conjunción con otras variables.

\section{b) En el ámbito del Management de la Calidad}

- Las escalas de calidad de servicio han de ser construidas de acuerdo con la finalidad que persigan. Si se trata de diagnosticar globalmente la calidad de servicio o de certificarla, quizás baste con la utilización de un indicador global de la misma (calidad de servicio global). Si se trata de diagnosticar y comparar en sus dimensiones principales, habrá que recurrir a las dimensiones resultantes en cada sector o tipo de empresas. Si se trata de buscar elementos concretos de mejora de calidad, es más recomendable la utilización de amplias listas de elementos, guarden o no relación con las dimensiones tradicionalmente consideradas.

- Respecto a las formas de operacionalizar el constructo 'calidad de servicio bancario', la sola consideración del desempeño es la fórmula más fiable y parsimoniosa, y , a la vez, la más rápida y económica en la captación y análisis dela información.

\section{BIBLIOGRAFIA}

ARBUCKLE, J.L. (1997): AMOS User's Guide (vers. 3.6). SmallWaters Corp.

BIGNÉ, SÁNCHEZ, MOLINER, VALLET. (1996): Fiabilidad y validez de los modelos de medición de calidad de servicio. Una aplicación a los servicios de transporte ferroviario. Ponencia en Congreso AEDEM, Granada.

CRONBACH, L.(1951): Coefficient Alpha and the Internal structure of Test. Psychometrica, $n^{\circ} 16$, Oct. 1951, 297-334.

CRONIN, J. J.; TAYLOR, Steven A. (1992): Measuring Service Quality: A Reexamination and Extension. Journal of Marketing,vol.56,n'1, 55-67.

CRONIN, J..; TAYLOR, S. (1994): SERVPERF versus SERVQUAL: Reconciling PerformanceBased and Perceeption-Minus-Expectations Measurement of Service Quality. Journal of Marketing, vol.58, n¹, 125-31.

JORESKOG, K., SORBOM, D. (1983): LISREL V and LISREL VI. Analysis of linnear structural relationship by maximum lekelihood and leats square methods. University of Upsala, Suecia.

JORESKOG, K., SORBOM, D. LISREL,Analysis of Linear Structural Relationships by the methods of Maximun Likelihood. III, 99-101, Uppsala, 1984.

PARASURAMAN, A.; ZEITHAML, V.A.; BERRY, L. (1985):_A coneptual model of service quality and its implications for future research. Journal of Marketing, vol. 49. 
Revista Eletrônica de Ciência Administrativa (RECADM) - ISSN 1677-7387

Faculdade Cenecista de Campo Largo - Coordenação do Curso de Administração v. 3, n. 2, nov./2004 - http://revistas.facecla.com.br/index.php/recadm/

PARASURAMAN, A., ZEITHAML, V., BERRY, L. (1986): SERVQUAL: A Multiple Item Scale for Measuring Customer Perception of Service Quality. Cambridge, Mass.:Marketing Science Institute. También en Journal of Retailing, vol. 64, $\mathrm{n}^{\circ} 1$, pp. 12-40.

PARASURAMAN, A.; ZEITHAML, V.A.; BERRY, L. (1994): Reassessment of Expectations as a Comparison Standar in Measuring Service Quality: Implications for Further Research. Journal of Marketing, vol.58, $\mathrm{n}^{0} 1$, 111-124.

SANTIAGO MERINO, José (1994): Calidad de Servicio en las Entidades Financieras: Variables y Medición. Encuentros de Profesores Universitarios de Marketing, Sevilla, Octubre.

SARIS W.E. Y STRONKHORST, L.H. (1984): Introduction to causal models in non-experimental research. Amsterdam. Sociometric Research Foundation, p. 265.

TEAS, R. KENNETH. (1993): Expectations, Performance Evaluation, and Consumer's Perceptions of Quality. Journal of Marketing, vol. 57, nº4, 18-34.

TEAS, R. KENNETH. (1994): Expectations as a Comparison Standar in Measuring Service Quality: An Assessment of a Reassessment. Journal of Marketing, vol.58, n¹, 132-39.

ZEITHAML, V., PARASURAMAN, A., BERRY, L. (1993): Calidad Total en la Gestión de servicios. Madrid. Diaz de Santos.

ZEITHAML, V.A.; BERRY, L.L.; PARASURAMAN. (1996): The Behavioral Consequences of Service Quality. Journal of Marketing, vol.1, april , p. 31

ZEITLHAML, PARASURAMAN, BERRY (1990): Delivering Quality Service. The Free Press.1990.

ANEXOS: 
Revista Eletrônica de Ciência Administrativa (RECADM) - ISSN 1677-7387

Faculdade Cenecista de Campo Largo - Coordenação do Curso de Administração

v. 3, n. 2, nov./2004 - http://revistas.facecla.com.br/index.php/recadm/

Figura nº 1: Factores Determinantes de la Calidad de Servicio Bancario

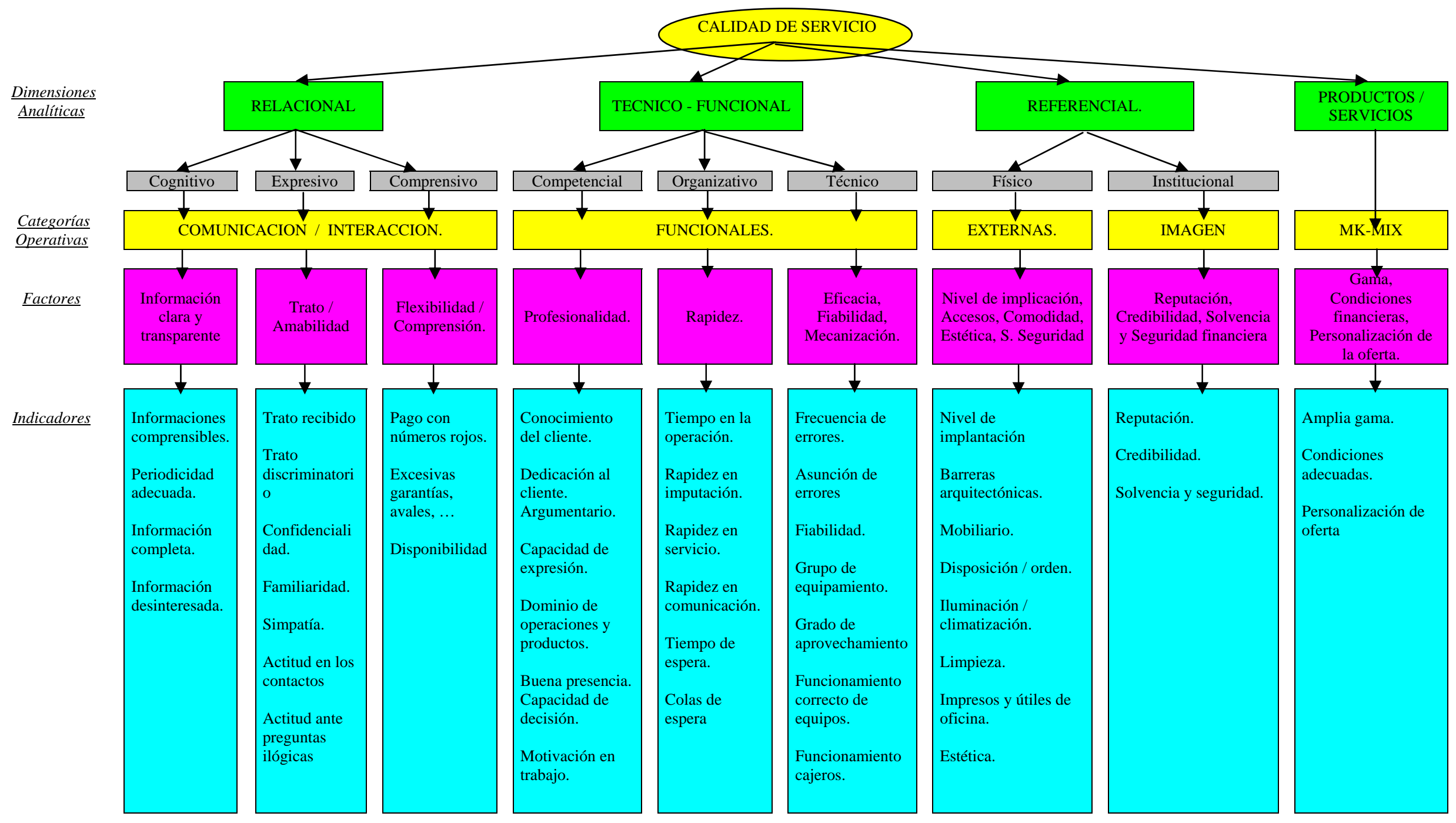


Revista Eletrônica de Ciência Administrativa (RECADM) - ISSN 1677-7387

Faculdade Cenecista de Campo Largo - Coordenação do Curso de Administração

v. 3, n. 2, nov./2004 - http://revistas.facecla.com.br/index.php/recadm/ 
Revista Eletrônica de Ciência Administrativa (RECADM) - ISSN 1677-7387

Faculdade Cenecista de Campo Largo - Coordenação do Curso de Administração

v. 3, n. 2, nov./2004 - http://revistas.facecla.com.br/index.php/recadm/

Tabla n 5 Factorial Exploratorio: Estructura - Dimensiones y Saturaciones

\begin{tabular}{|c|c|c|}
\hline Elementos & Saturación & Explicación - Contenido \\
\hline FACTOR I & $(19,97 \%)$ & Profesionalidad / Trato / Amabilidad \\
\hline CSR27 & .715 & Los empleados muestran buena disposición para atender al público \\
\hline CSR13 & .698 & Los empleados muestran una actitud cordial en el contacto inicial y la despedida \\
\hline CSR22 & ,693 & Los empleados saben escuchar al cliente \\
\hline CSR24 &, 686 & Los empleados parecen estar satisfechos con su trabajo y muestran interés por el mismo \\
\hline CSR26 & ,684 & Los empleados muestran esmero y celo profesional \\
\hline CSR14 & ,681 & El personal es cortés, paciente y no pierde los buenos modales aunque le hagan \\
\hline CSR11 &, 680 & Simpatía personal \\
\hline CSR15 & ,665 & Habilidad para conectar con el cliente \\
\hline CSR12 & ,661 & El personal concentra su atención en el cliente y sus asuntos \\
\hline CSR21 &, 650 & Los empleados tienen suficiente facilidad de expresión-dominio del vocabulario \\
\hline CSR7 & 649 & Trato respetuoso y cortés \\
\hline CSR19 & ,604 & Los empleados dedican todo el tiempo necesario a las gestiones con el cliente... \\
\hline CSR10 &, 522 & Familiaridad \\
\hline CSR25 &, 560 & Los empleados muestran una compostura-aspecto cuidados y actitud ordenada \\
\hline CSR1 &, 543 & Ante cualquier pregunta o duda los empleados dan explicaciones comprensibles \\
\hline CSR20 &, 538 & Los empleados ofrecen argumentos o razones para operar con la entidad o para... \\
\hline CSR23 &, 520 & Los empleados muestran un aceptable dominio de las operaciones, los temas, los \\
\hline CSR30 &, 506 & Diligencia mostrada por los empleados en gestiones con el cliente \\
\hline CSR9 &, 473 & En consultas y gestiones se guardan las elementales normas de discrección-intimidad \\
\hline CSR8 & ,467 & Trato no discriminatorio en función del volumen de negocio, sexo, edad... \\
\hline CSR18 & ,405 & Los empleados conocen la situación personal y financiera del cliente \\
\hline FACTOR II & $(9,58 \%)$ & Oficinas / Instalaciones \\
\hline CSR44 &, 765 & Mobiliario funcional y moderno en las oficinas \\
\hline CSR50 & ,727 & Estética agradable (interior y exterior, diseño y decoración) de la oficina \\
\hline CSR45 & ,726 & Aspecto cuidado y orden reinante en las oficinas \\
\hline CSR46 & ,687 & Limpieza correcta de las instalaciones \\
\hline CSR47 & ,601 & Separación de área de ventanillas y área de consulta y gestión \\
\hline CSR48 & ,587 & Impresos de operación, folletos de productos y útiles de escritura visibles y disponibles \\
\hline CSR43 &, 543 & Accesos desde la calle cómodos para el público (ausencia de barreras arquitectónicas) \\
\hline CSR49 & ,511 & Sistemas de seguridad (cristales blindados, guardas...) suficientes \\
\hline CSR38 & ,370 & Suficiente nivel de equipamiento informático-técnico \\
\hline CSR6 &, 296 & Publicidad en oficinas de los diversos tipos de interés y comisiones aplicados \\
\hline FACTOR III & $(5,88 \%)$ & Imagen / Productos / Servicios \\
\hline CSR54 &, 678 & Buena reputación de la entidad \\
\hline CSR55 &, 672 & Credibilidad \\
\hline CSR56 & 644 & Solidez y fiabilidad financiera \\
\hline CSR53 & ,554 & Condiciones financieras ventajosas: tipos de interés, plazos amortización, comisiones ... \\
\hline CSR52 &, 554 & Adaptación de los productos y servicios financieros ofrecidos a las necesidades del cliente \\
\hline CSR51 & ,516 & Amplia gama de productos y servicios para cubrir las necesidades del cliente \\
\hline FACTOR IV & $(5,21 \%)$ & Rapidez \\
\hline CSR34 & ,841 & Ausencia de personas en cola de espera para ser atendidas \\
\hline CSR35 & ,804 & Corto tiempo de espera desde que se entra hasta que se es atendido \\
\hline CSR39 &, 558 & Buen nivel de aprovechamiento de los equipos técnicos/ventanillas disponibles \\
\hline CSR31 &, 543 & $\begin{array}{ll}\text { Rapidez de servicio en la oficina } \\
\end{array}$ \\
\hline FACTOR V & $(4,98 \%)$ & Información / Transparencia \\
\hline CSR5 & ,726 & Claridad y nivel de comprensión adecuado de extractos y otras informaciones \\
\hline CSR4 & ,634 & Frecuencia adecuada en envío de extractos de cuenta y otra información complementaria \\
\hline CSR2 &, 572 & La información que recibe de la entidad es completa, con sus "pros" y sus "contras" \\
\hline CS37 & ,519 & Exactitud y claridad de las comunicaciones enviadas al cliente \\
\hline CSR8 &, 507 & Trato no discriminatorio en función del volumen de negocio, sexo, edad... \\
\hline CSR3 &, 443 & La información proporcionada está orientada en beneficio del cliente \\
\hline FACTOR VI & $(4,82 \%)$ & Eficacia/Fiabilidad \\
\hline CSR29 &, 666 & Rapidez de imputación de cargos y abonos \\
\hline CSR33 & .599 & Prontitud en la comunicación de los movimientos en cuentas \\
\hline CSR41 &, 565 & Fiabilidad: cumplen lo que prometen y hacen bien las cosas "a la primera" \\
\hline CSR32 & ,553 & Rapidez y agilidad en transacciones y operaciones informatizadas \\
\hline CSR36 & ,422 & Prontitud en la resolución de errores \\
\hline
\end{tabular}


Revista Eletrônica de Ciência Administrativa (RECADM) - ISSN 1677-7387

Faculdade Cenecista de Campo Largo - Coordenação do Curso de Administração v. 3, n. 2, nov./2004 - http://revistas.facecla.com.br/index.php/recadm/

\begin{tabular}{|c|c|c|}
\hline CSR28 &, 376 & Ausencia de errores humanos \\
\hline FACTOR VII (3,12\%) & Funcionamiento de Cajeros y Equipos \\
\hline CSR40 &, 653 & Correcto funcionamiento de los equipos técnicos instalados \\
\hline CSR42 &, 630 & Correcto funcionamiento y disponibilidad de fondos en cajeros automáticos \\
\hline FACTOR VIII & $(\mathbf{1 , 8 5 )} \%$ & Flexibilidad \\
\hline CSR17 &, 527 & Horario de atención al público suficientemente flexible \\
\hline CSR16 &, 459 & La entidad evita, en lo posible, excesivas garantías, avales y papeleo \\
\hline
\end{tabular}

Método de extracción: Análisis de componentes principales. Método de rotación: Normalización Varimax con Kaiser.

Tabla $n^{\circ} 6$ Fiabilidad y Varianza Explicada por formas alternativas de medición de la Calidad de Servicio.

\begin{tabular}{||c|c|c|c|c|c|c|c||}
\hline \multicolumn{2}{||c|}{} & \multicolumn{2}{|c|}{$\begin{array}{c}\text { Fiabilidad escala } \\
\text { total }\end{array}$} & \multicolumn{2}{c||}{$\%$ Varianza Explicada } \\
\hline $\begin{array}{c}\text { Dimensio } \\
\text { nes }\end{array}$ & $\begin{array}{c}\text { No } \\
\text { Items }\end{array}$ & $\begin{array}{c}\text { SERVQU } \\
\text { AL }\end{array}$ & $\begin{array}{c}\text { SERPR } \\
\text { EF }\end{array}$ & Sector & Autor & SERVQUAL & SERVPERF \\
\hline 4 & 17 & 0.92 & & Banca & $\begin{array}{l}\text { Necmi Kemal Avkiram } \\
(94)\end{array}$ & 54.3 & \\
\hline 1 & 22 & 0.89 & 0.92 & Banca & $\begin{array}{c}\text { Cronin y Taylor } \\
(92)\end{array}$ & 34.8 & 41.1 \\
\hline 5 & 22 & 0.76 & 0.77 & $\begin{array}{c}\text { Transp. } \\
\text { Públicos }\end{array}$ & Bigné et alia (98) & 51.3 & 58.2 \\
\hline 8 & 56 & & 0.96 & Banca & Santiago (99) & & 54.5 \\
\hline
\end{tabular}

Tabla n 7: Análisis factorial confirmatorio de la C.S.B.

\begin{tabular}{|c|c|c|c|c||}
\hline \multirow{2}{*}{ AMOS: Indices } & \multicolumn{4}{|c||}{ C.S.B. } \\
\cline { 2 - 5 } & 1 FACTOR & $\begin{array}{c}5 \\
\text { FACTORES }\end{array}$ & $\begin{array}{c}8 \\
\text { FACTORES }\end{array}$ & $\begin{array}{c}25 \\
\text { FACTORES }\end{array}$ \\
\hline Indice de Bondad de Ajuste (GFI) & 0,773 & 0,816 & 0,831 & 0,890 \\
\hline Indice de Ajuste Ajustado (AGFI) & 0,747 & 0,801 & 0,815 & 0,852 \\
\hline $\begin{array}{c}\text { Raiz del Residuo Cuadrado Medio } \\
\text { (RMR) }\end{array}$ & 0,055 & 0,288 & 0,061 & 0,043 \\
\hline \hline
\end{tabular}

Tabla n 8: Ajuste de modelos AMOS por método ULS

\begin{tabular}{||c|c|c|c|c|c||}
\hline MODELO & NPAR & CMIN & RMR & GFI & AGFI \\
\hline 1 FACTOR & 112 & 7493,7 & 0,063 & 0,964 & 0,961 \\
\hline 5 FACTORES & 121 & 12221 & 0,080 & 0,941 & 0,981 \\
\hline
\end{tabular}


Revista Eletrônica de Ciência Administrativa (RECADM) - ISSN 1677-7387 Faculdade Cenecista de Campo Largo - Coordenação do Curso de Administração v. 3, n. 2, nov./2004 - http://revistas.facecla.com.br/index.php/recadm/

\begin{tabular}{|c|c|c|c|c|c||}
\hline 8 FACTORES & 140 & 3912 & 0,045 & 0,981 & 0,979 \\
\hline 25 FACTORES & 402 & 2137,1 & 0,033 & 0,99 & 0,986 \\
\hline
\end{tabular}

Tabla n 9: Medidas de ajuste de los modelos de medición de la C.S.B.

\begin{tabular}{|c|c|c|c|c|c|}
\hline Modelo & Fallos $^{0}$ & $\begin{array}{c}\text { Discrepancia } \\
\text { media (s.e.) }\end{array}$ & BCC $^{1}$ & AIC $^{2}$ & CAIC $^{3}$ \\
\hline 1 FACTOR & 0 & $7220,16(1,00)$ & 7306,4 & 7289,6 & 8312,4 \\
\hline 5 FACTORES & 0 & $6736(0,87)$ & 6784,35 & 6772,25 & 7508,95 \\
\hline 8 FACTORES & 0 & $6173(0,92)$ & 6237,14 & 6223,14 & 7075,52 \\
\hline 25 FACTORES & 0 & $4512,2(0,46)$ & 4826,09 & 4782,5 & 7388,3 \\
\hline M. SATURADO & & & 3351,6 & 3192,0 & 12909,1 \\
\hline M. INDEPENDEN. & & & 33460,46 & 33454,8 & 33795,8 \\
\hline
\end{tabular}

1.BCC: Ajuste de Borwne-Cudek (1989).2.AIC: Criterio de Información de Akaike (1973, 1987).3.CAIC: AIC consistente; criterio de Bozdogan's (1987).

Tabla ${ }^{\circ}$ 10: Correlaciones Calidad Global con las diversas formas de operacionalizar el constructo C.S.B.

\begin{tabular}{|c|c|}
\hline Forma de Operacionalización & Correlación \\
\hline C.S. PERCIBIDA & $.474^{\star \star}$ \\
\hline C.S. PERCIBIDA x IMPORTANCIA & $.429^{\star \star}$ \\
\hline C.S.(PERCIBIDO-ESPERADO) & $.301^{\star \star}$ \\
\hline C.S.(PERCIBIDO-ESPERADO)x IMPORTANCIA & $.280^{\star *}$ \\
\hline
\end{tabular}

** = significativas al 0,01 (bilateral)

Tabla n 11: Coeficientes de Regresión con Calidad Global

\begin{tabular}{|c|c|c|c|c|}
\hline Forma de Operacionalización & $\begin{array}{c}\text { Coef. Beta No } \\
\text { estandarizados }\end{array}$ & $\begin{array}{c}\text { Coef. Beta } \\
\text { estandarizados }\end{array}$ & $\begin{array}{c}\text { Valor } \\
\text { "t" }\end{array}$ & Significación \\
\hline C.S. PERCIBIDA &, 770 &, 229 & 3,427 &, 001 \\
\hline $\begin{array}{c}\text { C.S.(PERCIBIDAxIMPORTANCIA } \\
\text { ) }\end{array}$ &, 0063 &, 155 & 2,050 &, 041 \\
\hline $\begin{array}{c}\text { C.S. (PERCIBIDO-ESPERADO) } \\
\text { CS.S.((PERCIBIDO- }\end{array}$ &,- 384 &,- 136 & $-1,032$ &, 302 \\
\hline ESPRADO)xIMPORTANCIA) &, 138 &, 213 & 1,604 &, 109 \\
\hline
\end{tabular}


Revista Eletrônica de Ciência Administrativa (RECADM) - ISSN 1677-7387 Faculdade Cenecista de Campo Largo - Coordenação do Curso de Administração v. 3, n. 2, nov./2004 - http://revistas.facecla.com.br/index.php/recadm/ 